\title{
1 Bacterial epibiont communities of panmictic Antarctic krill are spatially structured
}

2 Laurence J. Clarke ${ }^{1,2,3 *}$, Léonie Suter ${ }^{1}$, Rob King ${ }^{1}$, Andrew Bissett ${ }^{4}$, Sophie Bestley ${ }^{2}$, Bruce E. Deagle ${ }^{1,4}$ 3

$4 \quad{ }^{1}$ Australian Antarctic Division, Channel Highway, Kingston, Tasmania 7050, Australia

$5 \quad{ }^{2}$ Institute for Marine and Antarctic Studies, University of Tasmania, Hobart, Tasmania 7001, Australia

$6{ }^{3}$ Antarctic Climate \& Ecosystems Cooperative Research Centre, University of Tasmania, Hobart,

$7 \quad$ Tasmania 7001, Australia

$8{ }^{4}$ Commonwealth Scientific and Industrial Research Organisation, Hobart, Tasmania 7004, Australia 9

$10{ }^{*}$ Corresponding author: Laurence J. Clarke, Australian Antarctic Division, Kingston, Tasmania 7050,

11 Australia. Ph: +61 (3) 62323117

12 Email: laurence.clarke@utas.edu.au

13

Short title: Krill microbiomes are spatially structured

15 
Antarctic krill (Euphausia superba) are amongst the most abundant animals on Earth, with a circumpolar distribution in the Southern Ocean. Genetic and genomic studies have failed to detect any population structure for the species, suggesting a single panmictic population. However, the hyper-abundance of krill slows the rate of genetic differentiation, masking potential underlying structure. Here we use high-throughput sequencing of bacterial 16S rRNA genes to show that krill bacterial epibiont communities exhibit spatial structuring, driven mainly by distance rather than environmental factors, especially for strongly krill-associated bacteria. Estimating the ecological processes driving bacterial community turnover indicated this was driven by bacterial dispersal limitation increasing with geographic distance. Furthermore, divergent epibiont communities generated from a single krill swarm split between aquarium tanks under near identical conditions suggests physical isolation in itself can cause krill-associated bacterial communities to diverge. Our findings show that Antarctic krill-associated bacterial communities are geographically structured, in direct contrast with the lack of structure observed for krill genetic and genomic data.

\section{Introduction}

Antarctic krill (Euphausia superba) is a keystone species of Southern Ocean food webs (Croxall et al. 1999), with an estimated biomass of 379 million tonnes (Atkinson et al. 2009). The commercial Antarctic krill fishery catch is the largest in the Southern Ocean and has been expanding in recent years (Nicol \& Foster 2016). However, management of the krill fishery is hampered by lack of knowledge regarding krill population structure and the number of effective stocks.

Krill have a circumpolar, but non-uniform, distribution, with highest densities in the South Atlantic near the Antarctic Peninsula and Scotia Arc (Siegel \& Watkins 2016). The presence of persistent areas of high krill density has led to suggestions that there may be between three and six separate krill stocks 
and genomic studies over the last four decades (Deagle et al. 2015). The current consensus is that the genetic and genomic data are consistent with a single panmictic circumpolar population, however, any demographic structure may be obscured by the hyper-abundance of Antarctic krill (Jarman \& Deagle 2016). Genetic drift in neutral markers is inversely related to population size, and the vast number of individual krill would slow genetic differentiation between subpopulations, even in the absence of homogenising gene flow (Deagle et al. 2015).

All eukaryotes carry a characteristic bacterial microbiome. Bacterial populations often exhibit more genetic structuring than those of higher eukaryotes, as large bacterial populations accumulate diversity that tends to create barriers to recombination between lineages (Yang et al. 2019). Spatial structuring of microbiomes has been demonstrated in several marine invertebrates (Cregeen 2016; Zwirglmaier et al. 2015), and can arise as a result of either environmental (including host) heterogeneity combined with selection, and/or dispersal limitation combined with ecological drift (Stegen et al. 2015). In a recent study, we showed that four krill swarms separated by $260-1270 \mathrm{~km}$ each supported distinct microbiomes, with the strongest differences observed in bacteria associated with the exoskeleton (Clarke et al. 2019). In this study, we expand the number of swarms and the number of krill sampled per swarm, and examine a greater range of geographic distances to test whether krill-associated bacteria show spatial structuring, and whether the observed structure is more closely related to geographic or environmental distance. In supporting experiments, we investigate whether divergent krill microbiomes can be generated in aquaria under near identical conditions. Our results demonstrate krill microbiomes in different areas represent distinct communities, and may also be functionally different; these different microbe-host associations in different spatial areas may be meaningful as conservation units.

65 
We analysed the bacterial communities on 155 krill moults collected from krill from 13 swarms ( $n=11$ 12 moults per swarm) separated by $4-3481 \mathrm{~km}$ (Fig. 1a). Following filtering and quality control, this dataset included 4.49 million Illumina paired-end sequencing reads $(10,787$ to 87,547 reads per sample) representing 3,808 zero-radius OTUs (zOTUS). Moult bacterial communities were dominated by Gammaproteobacteria (mean relative abundance $53 \%$ ), followed by Bacteroidia $(27 \%$ ) and Alphaproteobacteria (7\%). Swarm membership (trawl) explained half the variance in moult microbiome composition based on weighted UniFrac distance, and more than $40 \%$ for unweighted UniFrac distance (weighted UniFrac: $F_{12,142}=11.85, R^{2}=0.500, P<0.001$, unweighted UniFrac: $F_{12,142}=8.18, R^{2}=0.409, P<0.001$ ). Ecological (unweighted UniFrac) distance between moult bacterial communities increased with increasing geographic distance between trawls (Fig. 2a). An UPGMA tree based on unweighted UniFrac distance showed bacterial communities clustered by geographic region (Fig. 1a and b), with 3-5 swarms sharing similar microbiomes near each of Mawson, Casey and Dumont d'Urville stations in East Antarctica. A single swarm near BANZARE Bank north-east of Mawson station (K29) supported a distinct microbiome, though most closely related to the swarms sampled off Mawson (Fig. 1b). The spatial structuring of krill microbiomes from the vicinity of Casey and Mawson is in direct contrast to the lack of structure observed in SNP markers for krill collected from the same two regions (Deagle et al. 2015) (Fig. 3).

Although we have previously demonstrated that krill moult bacterial communities are distinct from seawater bacterial communities (Clarke et al. 2019), moult bacterial communities are an open system that can exchange bacteria with the surrounding seawater. To investigate if variation in seawater bacteria could be driving the observed geographic structuring, we repeated the above analyses using only strongly krill-associated bacteria (present in $\geq 50 \%$ of samples) to reduce the potential influence of local environmental bacteria. This reduced the dataset from 3,808 to 267 zOTUs but, as these were the more common bacteria, still represented $73.3 \%$ of reads. The entire moult and strongly krillassociated bacterial communities were taxonomically very similar (see Supporting Information Text) 
and strongly krill-associated bacterial communities clustered by region as per Fig. 1b (Fig. S1). Swarm membership explained approximately half the variance in strongly krill-associated bacterial community composition (weighted UniFrac: $R^{2}=0.469$, unweighted UniFrac: $R^{2}=0.529, P<0.001$ for both). Unweighted UniFrac distance between strongly krill-associated bacterial communities also increased with geographic distance between trawls (Fig. S2a).

\section{Identity of dominant core zOTUs differs between regions}

The core microbiome (zOTUs present in $85 \%$ or more of the moult samples) comprised only 21 zOTUs representing $30.9 \%$ of reads. The core microbiome was dominated by zOTUs from the families Colwelliaceae (13 zOTUs), Rhodobacteraceae (4 zOTUs, 2 Sulfitobacter) and Flavobacteriaceae (3 zOTUS, 2 Polaribacter). Most of the Colwelliaceae zOTUs (11/13) had 97-99\% identity to two closely related (99\% identity) Colwellia species isolated from Antarctic sea-ice assemblages (Bowman et al. 1998) (Table S1). Other core zOTUs were highly similar (99-100\% pairwise identity) to bacterial isolates from polar marine invertebrates ( $n=3$ zOTUs) and to Antarctic sea ice bacteria ( $n=3$ zOTUs), indicating these high nutrient surface environments in polar oceans are commonly colonised by similar bacterial assemblages. Interestingly, despite being present in the majority of samples and representing a high proportion of the total reads, the identity of dominant core zOTUs varied between geographic regions

(Fig. 4). Four core zOTUs (three Colwellia and one Sulfitobacter) were identified as having significantly different relative abundances between regions (LEfSe, LDA $>3, \alpha<0.01$ ). Inferred metagenomic traits also suggest krill moult bacterial communities from different regions are functionally distinct (Fig. S3 and 4).

\section{Environmental and spatial effects}

We used linear mixed-effects (LME) modelling to test whether spatial (geographic distance) and/or environmental variables (ocean temperature, salinity, sea-ice melt and productivity) explained variation in krill microbial community composition between trawl sites. Geographic distance (LME 
model coefficient estimate: $0.065 \pm 0.003[0.060-0.07095 \% \mathrm{Cl}])$ and salinity $(0.009 \pm 0.003[0.003-0.014$ $95 \% \mathrm{Cl}]$ ) were the only significant predictors for moult community composition $(z=23.01, p<0.001$ and $z=3.19, p=0.0014$, respectively, Table S4), based on model-averaging over the top-ranked models $(n=8)$ using $\triangle \mathrm{AICc} \leq 10$. In contrast, geographic distance $(0.066 \pm 0.003[0.059-0.07395 \% \mathrm{Cl}])$ was the only significant predictor for strongly krill-associated bacterial communities $(z=18.8, p<$ 0.001, Table S6), with weaker evidence for an effect of salinity depending on the random-effects specification (custom covariance structure: $z$-value $=1.48, p$-value $=0.14$, Table S6; crossed effects zvalue $=2.21, p$-value $=0.027$, Table S10). Results were very consistent overall using the two different random-effects specifications (Table S3-10). All top-ranking (i.e., lower AICc) models included and strongly krill-associated bacteria.

\section{Krill microbiome differentiation driven by dispersal limitation over large geographic distances}

We used a combination of null models to estimate the contribution of homogeneous or variable selection, homogenising dispersal and dispersal limitation to krill microbiome community turnover (Stegen et al. 2013; Stegen et al. 2015). In our data, bacterial communities on krill with increased geographic separation showed increased dispersal limitation and decreased influence of homogenising dispersal, homogeneous selection and undominated processes. Undominated processes were the major influence for moult communities sampled from krill within a trawl (52\%, Fig. 2b). Comparing samples between trawls within a cluster (e.g., Mawson, Casey and Dumont d'Urville), the influence of dispersal limitation increased from 9 to $29 \%$, with decreased influence of 140 homogenising dispersal and undominated processes. Dispersal limitation was the main factor when 141 comparing communities between clusters (57\%), with a further decrease in the influence of 142 undominated processes (18\%). The contribution of homogeneous selection and homogenising dispersal also decreased from 11 to $2 \%$ and 11 to $0.3 \%$, respectively, with increasing geographic 
separation. Strongly krill-associated bacteria showed similar patterns of increasing dispersal limitation with increasing geographic distance (Fig. S2b).

To investigate the process of microbiome differentiation, krill from a single swarm off Casey station (T04, see Fig. 1a) were split across four $200 \mathrm{~L}$ aquarium tanks, with approximately 400 krill per tank, and with consistent conditions for seven months prior to sampling. Krill from each tank developed significantly different epibiont bacterial communities, with twice the proportion of variation explained by tank of origin when relative abundance was taken into account, compared to presence/absence of taxa (weighted UniFrac (WU): $F_{3,44}=12.09, R^{2}=0.452, P<0.001$, unweighted UniFrac (UU): $F_{3,44}=4.57$, $R^{2}=0.237, P<0.001$, Fig. 5a and b). Bacterial communities in water within the four tanks were clearly distinct from krill communities (Fig. S5, WU: $F_{1,54}=46.56, R^{2}=0.463, P<0.001$, UU: $F_{1,54}=11.91, R^{2}=0.181$, $P<0.001$ ), but were also significantly different between tanks (WU: $F_{3,4}=2.87, R^{2}=0.682, P=0.015, \mathrm{UU}$ : $\left.F_{3,4}=1.65, R^{2}=0.553, P=0.024\right)$. However, this was largely driven by tank $2 \mathrm{~A}$, as tank water bacterial communities were not significantly different when this tank was excluded $\left(2 \mathrm{~B}, \mathrm{C}\right.$ and $\mathrm{D}, \mathrm{WU}: F_{2,3}=2.78$, $R^{2}=0.650, P=0.13$, UU: $\left.F_{2,3}=1.56, R^{2}=0.510, P=0.067\right)$, whereas epibiont communities were distinct (WU: $F_{2,33}=11.50, R^{2}=0.411, \cup U: F_{2,33}=4.91, R^{2}=0.229, P<0.001$ for both).

Comparing the ecological processes contributing to community turnover within versus between tanks, the contribution of homogenising dispersal decreased from 17 to less than $1 \%$, whereas dispersal limitation increased from 9 to $20 \%$ (Fig. 5c). Interestingly, the influence of variable selection (reflecting different environments) decreased from $7 \%$ within tanks to $1 \%$ between tanks, supporting the notion that the tanks represented highly similar environments. 


\section{Discussion}

We show that Antarctic krill-associated bacterial communities are geographically structured, in direct contrast with the lack of structure observed for krill genetic and genomic data (Deagle et al. 2015). Individual taxa and communities within the human microbiome can be used as markers of migration and ancestry (Dominguez-Bello \& Blaser 2011; Henne et al. 2014), allowing resolution of migration events previously indistinguishable with human genetic markers (Moodley et al. 2009; Wirth et al. 2004). The potential for members of the human microbiome to accurately trace human migrations is dependent on the degree to which they are vertically transmitted (Dominguez-Bello \& Blaser 2011). In contrast, structuring of krill-associated bacterial communities is driven by homogenising dispersal within a krill swarm (horizontal transmission), but increasing dispersal limitation with increasing geographic distance (Fig. 2). Our findings support the conclusion from a study of bat fur microbiomes (Kolodny et al. 2018) that for species experiencing high levels of homogenising dispersal between individuals, the colony or swarm rather than the individual may be the meaningful biological unit on which selection influences the microbiome. Krill microbiome dynamics can thus be understood best in light of metacommunity theory, being influenced by both processes within the community (e.g., competition) and dispersal between communities (Burns et al. 2017; Miller et al. 2018).

The significant effect of spatial distance on both moult and strongly krill-associated bacterial communities, but limited evidence for environmental influence on the latter suggests different environments are not essential for the development of distinct bacterial communities. This is further supported by development of divergent microbiomes in aquarium krill populations under nearidentical conditions. The contribution of variable selection, reflecting community differences arising due to selection in different environments, showed a modest increase comparing bacterial communities within a swarm versus those in separate bacterial geographic clusters (17 to $21 \%$ for entire moults communities, 23 to $26 \%$ for strongly krill-associated bacteria). In contrast, the contribution of dispersal limitation showed a much larger increase from 9 to 57\% (Fig. 2b). If 
environmental factors were driving variation in krill microbiomes, we would expect swarms in different water masses to have distinct bacterial communities, as the Southern Ocean fronts separate waters with distinct physical and chemical properties. However, the Casey and Dumont d'Urville clusters include swarms north and south of one or more oceanographic fronts (Fig. 1a). Similarly, LEfSe analysis showed that swarms north or south of the southern boundary of the Antarctic Circumpolar Current were not enriched in particular zOTUs (Table S11). Rather, clusters were mainly distributed west to east rather than north to south, consistent with meso-scale circulation in the region. In particular, Mawson (and BANZARE Bank) clusters correspond to the Prydz Bay gyre, and the Casey cluster corresponds to recirculation within the Australian-Antarctic basin (Bindoff et al. 2000). A series of cyclonic eddies off the Adelie Coast that terminates near $130^{\circ} \mathrm{E}$ may link krill swarms in the Dumont d'Urville cluster, whilst isolating them from sites further west (Aoki et al. 2007). However, the influence of circulation patterns, environmental factors and variable selection on krill microbiomes warrants further investigation. The difference observed for aquarium populations between distance measures including or excluding relative abundance suggests ecological drift may be driving divergence in isolated populations in the absence of environmental selection. Weighted UniFrac distance (including relative abundance) explained twice the proportion of variation between tanks compared to unweighted UniFrac distance (a presence/absence metric), suggesting changes in relative abundance precede changes in community membership.

Ecological drift in krill epibiont communities is likely exacerbated by the regular moulting of the exoskeleton, which occurs every 12-30 days during summer (Reiss 2016). Characterising bacteria from moulted exoskeletons means samples represent bacterial communities at the same stage of the moult cycle. However, this was not the case for the aquarium samples. The occurrence of ciliate epibionts decreases from $66 \%$ in pre-moult individuals to $0 \%$ in post-moult krill (Tarling \& Cuzin-Roudy 2008), with bacterial epibionts likely to experience a similar, but potentially less extreme, drop in abundance. 
following the "competitive lottery model" (Burke et al. 2011; Hubbell 2001), although the presence of protozoan epibionts could also influence bacterial moult community composition. The existence of a core microbiome is consistent with the need for species to have similar ecologies to compete for the same vacant niche. Given krill swarms can exceed densities of 1000 individuals $\mathrm{m}^{-3}$ (Nowacek et al. 2011; Tarling et al. 2009), colonisation is most likely to occur from epibionts on neighbouring krill, hence the greater influence of homogenising dispersal for bacterial communities within a swarm (Fig. 2b). Homogenising dispersal should have less influence in smaller or less dense swarms, leading to faster rates of drift. Although bacteria on model marine (chitin) particles exhibit rapid and reproducible succession patterns (Datta et al. 2016), further aquarium studies are required to better understand krill epibiont colonisation dynamics.

The fishery for Antarctic krill is the largest by tonnage in the Southern Ocean (Nicol \& Foster 2016). The krill fishery is managed using regional catch limits, but whether there is a single circumpolar krill stock or if there are distinct populations within each region is unclear (Jarman \& Deagle 2016). We have shown that krill microbiomes in different regions represent distinct communities, indicating a non-homogeneous mixture throughout their range. Microbiomes may also be functionally different and furthermore affect host fitness. It seems plausible that different microbe-host associations in different regions may be meaningful as conservation units. Current spatial management of the krill fishery is focussed within the southwest Atlantic, where small-scale management units have been established. Our results suggest that the Indian Sector of the Southern Ocean contains at least understand how to apply microbe-host associations to inform regional krill fishery management. 
There are many ways population connectivity can be measured; from ancient linkages through phylogeography, to more recent linkages with population genetics, and contemporary movement though animal tracking. Several studies have now demonstrated links between host population genetic structure and host-associated microbiome composition in marine species (Díez-Vives et al. 2020; Easson et al. 2020; Fietz et al. 2018). Our study does not explicitly test whether there is a direct association between krill moult microbiomes and krill population dynamics; indeed this is not possible given that we have little independent information about krill population dynamics. However, we consider that some link between krill microbiomes and population connectivity is a reasonable hypothesis given the apparent lack of an environmental driver and observed isolation by distance. Future studies should explore and ideally test whether there is an association between krill microbiomes and krill population dynamics, especially in the key krill fishery areas of the Antarctic Peninsula and Scotia Arc. Supporting aquarium experiments that investigate horizontal transmission rates between krill with distinct microbiomes, and between krill and the water column, would also assist establishing the scope of inference.

260

The potential for long-distance dispersal in many marine taxa leads to high population connectivity, making it difficult to resolve population structure with classic genetic approaches (Kelley et al. 2016). An improved view of host-microbiome structuring could expand our understanding of system connectivity in the marine environment. Studying the structure of the bacterial communities that effectively hitch a ride with their host may allow previously unseen linkages and barriers to dispersal to be identified.

\section{Materials and Methods}

\section{Sample collection}

270 Samples were collected on board the RSV Aurora Australis during voyage 3 between 31 January and 27119 February 2016 (Kerguelen Axis voyage), and voyage 2 between 16 December 2016 and 16 January 
2017 (Totten Glacier voyage). Antarctic krill were sampled from 13 swarms across the Indian sector of the Southern Ocean (Fig. 1a) using targeted trawls with a Rectangular Mid-water Trawl 8+1 (RMT-8+1 metre square) net. Trawls were made on acoustically identified targets at depths between the surface and 60-70 $\mathrm{m}$. The pairwise distance between trawls ranged from 4 to $3481 \mathrm{~km}$.

In order to isolate moults, live krill were transferred immediately after capture to $250 \mathrm{~mL}$ jars (one krill per jar) which were ventilated with small holes to allow seawater exchange as per Virtue et al. (2010). The jars were incubated in a large (1600 L) flow-through seawater tank close to ambient ocean temperature (approx. $1{ }^{\circ} \mathrm{C}$ ) with no additional food provided (Kawaguchi et al. 2006). Jars were inspected for moults at 12 hour intervals, with the first 12 animals to moult from each trawl sampled for microbial community profiles (all collected within 48 hours). Moults were removed from the jar, rinsed with $0.22 \mu \mathrm{m}$-filtered seawater and stored separately in liquid nitrogen before being stored at $-86^{\circ} \mathrm{C}$ on return to Australia.

\section{Aquarium samples}

Krill from a single swarm caught off Casey station mid-December 2016 (T04 in Fig. 1a) were split across four 200 L krill aquaria tanks at the end of January 2017, with approximately 400 krill per tank. Krill in each tank experienced identical conditions for seven months, including light environment, food (phytoplankton), with the same filtered and UV-sterilised water supply for each tank (Kawaguchi et al. 2010; King et al. 2003). In September 2017, 12 krill per tank were individually netted and both lateral surfaces swabbed with Epicentre Catch-All ${ }^{\mathrm{TM}}$ sample collection swabs for approximately 10 seconds. Krill were then sexed and staged. Swabs were transferred to sterile Eppendorf tubes and stored at $86{ }^{\circ} \mathrm{C}$. Nets were treated with $1 \%$ bleach then $0.5 \%$ sodium thiosulphate and rinsed with reverseosmosis and deionised water between individuals. Tank water bacterial communities were sampled by filtering 1 to $1.6 \mathrm{~L}$ onto $0.22 \mu \mathrm{m}$ Sterivex $^{\mathrm{TM}}$ filters, with two samples per tank. Filters were transferred to tubes prior to extraction. 
Swabs $(n=48)$ were sent to the Australian Genome Research Facility (AGRF, Adelaide, Australia; Spectrophotometer (ThermoFisher Scientific). PCR amplification, amplicon purification and highthroughput sequencing of bacterial 16S V1-3 rRNA (primers 27F: AGAGTTTGATCMTGGCTCAG, Lane 1991; and 519R: GWATTACCGCGGCKGCTG, Lane et al. 1985) were carried out at the Ramaciotti Centre for Genomics (Sydney, Australia) on an Illumina MiSeq following the Australian Marine Microbes protocol (Brown et al. 2018).

\section{Data analysis}

DNA sequence processing and taxonomic assignment followed the Australian Marine Microbial Biodiversity Initiative workflow (Brown et al. 2018), with data presented as amplicon sequence variants, or zero-radius operational taxonomic units (zOTUs, Edgar 2016), to maximise potential phylogenetic resolution. In brief, paired-end reads were merged, short sequences ( $<400$ base pair, bp) and sequences containing $\mathrm{N}$ 's or homopolymer runs $>8$ bp were removed. Sequences were dereplicated and those with $<4$ representatives removed. Chimeras were removed and zOTUs identified using the UNOISE3 algorithm(Edgar 2016). Quality-filtered sequences (including those <4 representatives) were mapped to the zOTUs to create a sample-by-read abundance matrix. Taxonomy was assigned to each zOTU using the RDP Bayesian classifier (Wang et al. 2007) based on the SILVA v132 database (Yilmaz et al. 2014) and 60\% probability cut-off. Lastly, zOTUs present in only one sample or with less than 10 reads across the dataset were removed. The number of reads per sample was $10,787-87,547$ for moults, $13,083-72,384$ reads for swabs, and 5,107-10,215 for the Sterivex 
each sample type (Fig. S6). DNA sequence data for this study can be found in the NCBI database under BioProject ID: PRJNA505226. The zOTU table and mapping file are available on the Dryad data repository (Clarke et al. 2020).

Differences in entire moult and strongly krill-associated (present in $\geq 50 \%$ of samples) bacterial community composition between swarms were explored using weighted and unweighted UniFrac distances (Lozupone \& Knight 2005) in QIIME v1.8.0 (Caporaso et al. 2010) (beta_diversity_through_plots.py) based on a rarefied zOTU table $(10,000$ reads or 5,000 reads for entire moult and strongly krill-associated bacteria, respectively), with strength and significance of swarm assessed using the Adonis method (Anderson 2001) (compare_categories.py, 999 permutations). The same method was used to characterise differences between krill microbiomes from separate aquarium tanks, with the zOTU table rarefied to 5,000 reads for analyses including the Sterivex filters. The phylogenetic tree used for the UniFrac analysis and to estimate the contribution of selection and dispersal processes to microbiome turnover (see below) was generated in QIIME using PyNAST (Caporaso et al. 2009) to align sequences against the Greengenes (v13_8) core set (McDonald et al. 2012), then filtering the alignment (removing $0.0005 \%$ most variable positions and those that were $>80 \%$ gaps) and building the tree using FastTree 2.1.3 (Price et al. 2010).

We defined core moult microbiome membership as zOTUs present in $85 \%$ or more moult samples (>131/155 samples) using QIIME (compute_core_microbiome.py). Ecological dissimilarity between krill moult bacterial communities was visualised by an UPGMA tree based on unweighted UniFrac distance created using the jackknifed_beta_diversity.py workflow in QIIME, with subsampling repeated 100 times. The Linear Discriminant Analysis (LDA) Effect Size (LEfSe, Segata et al. 2011) method was used to highlight zOTUs that showed different abundances between bacterial geographic clusters (Fig. 1). Default settings were used except the LDA threshold was increased to 3.0 and the $\alpha$ value reduced to 0.01 to highlight the most significant taxa discriminating between clusters. 
We tested whether spatial (geographic distance) and/or environmental variables explained variation modelling approach (R package 'Ime4', Bates et al. 2015). Analyses were performed separately for both the entire moult community and strongly krill-associated bacteria. The response variable was of pairwise datasets (Clarke et al. 2002; Row et al. 2017) by setting up the covariance structure such that a proportion $\left(\rho_{\tau}\right)$ of the total variance $\left(\sigma^{2}\right)$ is due to the correlation between data points that share a common site. Thus, the covariance for $n$ pairwise data points that share a common site is $\rho_{\tau} \sigma^{2}$ and zero for those that do not (Clarke et al. 2002; Van Strien et al. 2012). These results were compared against LME models that more straightforwardly specified the two trawl sites as crossed random effects.

Predictor variables considered as fixed-effects included geographic distance $(\mathrm{km})$ between trawl sites and a suite of environmental variables obtained from both ship-based underway (sea surface temperature $\left[{ }^{\circ} \mathrm{C}\right]$ and salinity [on the practical salinity scale, PSS]) and satellite-derived data (time data are given in the Supporting Information. Euclidean distances were calculated for all environmental variable pairs between sites. We centred and scaled all predictor variables and ensured that the maximum correlation between predictor variables (all Pearson correlations $<0.66$ ) and variance inflation factors (all VIFs $\leq 1.52$ ) were acceptably low prior to model fitting (Zuur et al. 2010).

We adopted a multi-model inference and model averaging approach (Burnham \& Anderson 2002; Burnham et al. 2011) using the R package 'MuMIn' (Barton 2019) to generate a set of models with all combinations of fixed-effects. We calculated the marginal $\left(R_{\mathrm{m}}{ }^{2}\right)$ and conditional $\left(R_{\mathrm{c}}{ }^{2}\right)$ coefficient of 
determination (Nakagawa \& Schielzeth 2013) for all models, and for model evaluation used the second-order Akaike Information Criterion (AICC) suitable for small sample sizes, ranking the models via Akaike weights. For model averaging we included all models with a $\Delta \mathrm{AICc} \leq 10$ from the top-ranked model. We calculated the $95 \%$ confidence interval $(\mathrm{Cl})$ of the model-averaged regression coefficients (full average) and considered those which did not straddle zero to have a significant effect on the response under investigation. Results are presented in text as LME coefficient estimate $\pm \mathrm{SE}[95 \% \mathrm{CI}]$.

We used the procedures described by Stegen et al. $(2013 ; 2015)$ to estimate the contribution of selection and dispersal processes to krill microbiome assembly. Variable selection generates divergent communities due to distinct selective environments, whereas homogeneous selection generates convergent communities due to their presence in similar selective environments. Similarly, divergent communities arising primarily due to low rates of dispersal, which causes communities to drift apart, is referred to as 'dispersal limitation', whereas high dispersal preventing drift is referred to as 'homogenising dispersal'. The fraction of community turnover where neither selection nor dispersal is the primary driver of community turnover (e.g., due to the combination of moderate dispersal and weak selection) is referred to as 'undominated' (called 'drift' in Stegen et al. 2013; Stegen et al. 2015).

Firstly, we computed the between-community mean nearest taxon distance ( $\beta M N T D$ ), the mean phylogenetic distance between each zOTU in one community and its closest relative in a second community. A null-model distribution of this parameter was generated by randomly shuffling zOTUs across the tips of the phylogeny (999 permutations). $\beta$-nearest taxon indices ( $\beta N T I)$ were calculated as the difference between the observed $\beta M N T D$ and the mean of the null distribution, expressed in units of standard deviations; $\beta N$ TI values $<-2$ or $>+2$ were deemed significant deviations and indicative of homogeneous selection or variable selection, respectively. Where pairwise comparisons showed $|\beta N T I|<2$, we used the Bray-Curtis-based Raup-Crick metric (Chase et al. 2011; Stegen et al. 2013) to compare observed and expected turnover without using phylogenetic information to infer the 
contribution of homogenising dispersal, dispersal limitation, or undominated processes (moderate dispersal and weak selection, referred to as 'drift' in Stegen et al. 2013). For pairwise comparisons where $|\beta N T I|<2, R C_{\text {bray }}<-0.95$ represented homogenising dispersal, $\mathrm{RC}_{\text {bray }}>+0.95$ represented dispersal limitation, and $\mathrm{RC}_{\text {bray }}<|0.95|$ suggested no single ecological process dominated compositional turnover. We compared the relative contribution of each process for pairwise comparisons of krill bacterial communities within a swarm, between swarms within a bacterial geographical cluster (see Fig. 1), and between swarms in separate clusters; and within and between tanks for the aquarium populations.

\section{Data Acessibility}

DNA sequence data for this study can be found in the NCBI database under BioProject ID: PRJNA505226.

\section{Acknowledgements}

We thank the Science Technical Support Team of the Australian Antarctic Division (AAD) and the master and crew of the Aurora Australis for their considerable efforts to make this work happen. Jessica Melvin (U. Tasmania) collected samples during the Totten Glacier Voyage. Michael Sumner (AAD) helped extract the satellite-derived environmental data. Chris Burridge (U. Tasmania) gave advice on mixed-effect modelling. Stephen Nicol provided valuable discussions and comments on an earlier version of this manuscript. The manuscript was improved by insights provided by two anonymous reviewers. Molecular work was funded through the Australian Antarctic Science Program (AAS-4015 and 4313) and a Bioplatforms Australia Industry Access Voucher. LS was supported by an Early Postdoc Mobility fellowship from the Swiss National Science Foundation (P2EZP3_162241). SB is supported under an Australia Research Council Discovery Early Career Researcher Award (DE180100828). This work was a contribution to the Australian Antarctic Science Kerguelen Axis 
project (AAS-4344) and was supported by the Australian Government's Business Cooperative Research

428 Centres Programme through the Antarctic Climate and Ecosystems Cooperative Research Centre.

429

430 Funding statement

431 This work is supported by the Australian Antarctic Science Program (AAS-4015 and 4313 to BED), a

432 Bioplatforms Industry Access Voucher (to BED and LJC), an Australia Research Council Discovery Early

433 Career Researcher Award (DE180100828 to SB), and an Early Postdoc Mobility fellowship from the

434 Swiss National Science Foundation (P2EZP3_162241 to LS). The funders had no role in study design,

435 data collection and analysis, decision to publish, or preparation of the manuscript. 
Anderson MJ (2001) A new method for non-parametric multivariate analysis of variance. Austral Ecol. 26, 32-46.

Aoki S, Fukai D, Hirawake T, et al. (2007) A series of cyclonic eddies in the Antarctic Divergence off Adélie Coast. J. Geophys. Res. 112, C05019.

Atkinson A, Siegel V, Pakhomov EA, Jessopp MJ, Loeb V (2009) A re-appraisal of the total biomass and annual production of Antarctic krill. Deep-Sea Res. Pt. 1 Oceanogr. Res. Pap. 56, 727-740.

Avena CV, Parfrey LW, Leff JW, et al. (2016) Deconstructing the bat skin microbiome: influences of the host and the environment. Front. Microbiol. 7, 1753.

Barton K (2019) MuMIn: Multi-Model Inference. $R$ package version 1.43.6. https://CRAN.Rproject.org/package=MuMIn

Bates D, Mächler M, Bolker B, Walker S (2015) Fitting Linear Mixed-Effects Models UsingIme4. Journal of Statistical Software 67, 1-48.

Bindoff NL, Rosenberg MA, Warner MJ (2000) On the circulation and water masses over the Antarctic continental slope and rise between 80 and $150^{\circ}$ E. Deep-Sea Res. Pt. II. Top. Stud. Oceanogr. 47, 2299-2326.

Bowman JP, Gosink JJ, McCammon SA, et al. (1998) Colwellia demingiae sp. nov., Colwellia hornerae sp. nov., Colwellia rossensis sp. nov. and Colwellia psychrotropica sp. nov.: psychrophilic Antarctic species with the ability to synthesize docosahexaenoic acid (22:6w3). Int. J. Syst. Bacteriol. 48, 1171-1180.

Brown MV, van de Kamp J, Ostrowski M, et al. (2018) Systematic, continental scale temporal monitoring of marine pelagic microbiota by the Australian Marine Microbial Biodiversity Initiative. Sci. Data. 5, 180130.

Burke C, Steinberg P, Rusch D, Kjelleberg S, Thomas T (2011) Bacterial community assembly based on functional genes rather than species. Proc. Natl. Acad. Sci. USA 108, 14288-14293.

Burnham KP, Anderson DR (2002) Model Selection and Multimodel Inference: A Practical InformationTheoretical Approach., 2nd edn. Springer-Verlag, New York.

Burnham KP, Anderson DR, Huyvaert KP (2011) AIC model selection and multimodel inference in behavioral ecology: some background, observations, and comparisons. Behavioral Ecology and Sociobiology 65, 23-35.

Burns AR, Miller E, Agarwal M, et al. (2017) Interhost dispersal alters microbiome assembly and can overwhelm host innate immunity in an experimental zebrafish model. Proc. Natl. Acad. Sci. USA 114, 11181-11186.

Caporaso JG, Bittinger K, Bushman FD, et al. (2009) PyNAST: a flexible tool for aligning sequences to a template alignment. Bioinformatics 26, 266-267.

Caporaso JG, Kuczynski J, Stombaugh J, et al. (2010) QIIME allows analysis of high-throughput community sequencing data. Nat. Methods 7, 335-336.

Chase JM, Kraft NJB, Smith KG, Vellend M, Inouye BD (2011) Using null models to disentangle variation in community dissimilarity from variation in $\alpha$-diversity. Ecosphere $\mathbf{2}$, art24.

Clarke L, Suter L, King R, et al. (2020) Data for manuscript "Bacterial epibiont communities of panmictic Antarctic krill are spatially structured". Dryad, dataset, DOI: .

Clarke L, Suter L, King R, Bissett A, Deagle BE (2019) Antarctic krill are reservoirs for distinct Southern Ocean microbial communities. Front. Microbiol. 9, 3226.

Clarke RT, Rothery P, Raybould AF (2002) Confidence limits for regression relationships between distance matrices: Estimating gene flow with distance. Journal of Agricultural, Biological, and Environmental Statistics 7, 361-372.

Cregeen SJJ (2016) Microbiota of dominant Atlantic copepods: Pleuromamma sp. as a host to a betaproteobacterial symbiont Doctoral thesis, University of Southampton.

Croxall JP, Reid K, Prince PA (1999) Diet, provisioning and productivity responses of marine predators to differences in availability of Antarctic krill. Mar. Ecol. Prog. Ser. 177, 115-131. 
Datta MS, Sliwerska E, Gore J, Polz MF, Cordero OX (2016) Microbial interactions lead to rapid microscale successions on model marine particles. Nat Commun 7, 11965.

Deagle BE, Faux C, Kawaguchi S, Meyer B, Jarman SN (2015) Antarctic krill population genomics: apparent panmixia, but genome complexity and large population size muddy the water. Mol. Ecol. 24, 4943-4959.

Díez-Vives C, Taboada S, Leiva C, et al. (2020) On the way to specificity - Microbiome reflects sponge genetic cluster primarily in highly structured populations. Mol. Ecol., DOI: 10.1111/mec.15635.

Dominguez-Bello MG, Blaser MJ (2011) The human microbiota as a marker for migrations of individuals and populations. Annu. Rev. Anthropol. 40, 451-474.

Easson CG, Chaves-Fonnegra A, Thacker RW, Lopez JV (2020) Host population genetics and biogeography structure the microbiome of the sponge Cliona delitrix. Ecol. Evol. 10, 20072020.

Edgar RC (2016) UNOISE2: improved error-correction for Illumina 16S and ITS amplicon sequencing. bioRxiv, 081257.

Fietz K, Hintze COR, Skovrind M, et al. (2018) Mind the gut: genomic insights to population divergence and gut microbial composition of two marine keystone species. Microbiome 6, 82 .

Henne K, Li J, Stoneking M, et al. (2014) Global analysis of saliva as a source of bacterial genes for insights into human population structure and migration studies. BMC Evolutionary Biology 14, 190.

Hubbell SP (2001) The unified neutral theory of biodiversity and biogeography. Princeton University Press, Princeton.

Jarman SN, Deagle BE (2016) Genetics of Antarctic Krill. In: Biology and Ecology of Antarctic Krill. (ed. Siegel V), pp. 247-277. Springer, Cham.

Kawaguchi S, Candy SG, King R, Naganobu M, Nicol S (2006) Modelling growth of Antarctic krill. I. Growth trends with sex, length, season, and region. Mar. Ecol. Prog. Ser. 306, 1-15.

Kawaguchi S, King R, Meijers R, et al. (2010) An experimental aquarium for observing the schooling behaviour of Antarctic krill (Euphausia superba). Deep-Sea Res. Pt. II. Top. Stud. Oceanogr. 57, 683-692.

Kelley JL, Brown AP, Therkildsen NO, Foote AD (2016) The life aquatic: advances in marine vertebrate genomics. Nat. Rev. Genet. 17, 523-534.

King R, Nicol S, Cramp P, Swadling KM (2003) Krill maintenance and experimentation at the Australian Antarctic Division. Mar. Freshw. Behav. Physiol. 36, 271-283.

Kolodny O, Weinberg M, Reshef L, et al. (2018) Coordinated change at the colony level in fruit bat fur microbiomes through time. Nat. Ecol. Evol. 3, 116-124.

Lane DJ (1991) 16S/23S rRNA sequencing. In: Nucleic acid techniques in bacterial systematics (eds. Stachebrandt E, Goodfellow M), pp. 115-175. John Wiley and Sons, Chichester, NY.

Lane DJ, Pace B, Olsen GJ, et al. (1985) Rapid determination of 165 ribosomal RNA sequences for phylogenetic analyses. Proc. Natl. Acad. Sci. USA 82, 6955-6959.

Lankau EW, Hong PY, Mackie RI (2012) Ecological drift and local exposures drive enteric bacterial community differences within species of Galapagos iguanas. Mol. Ecol. 21, 1779-1788.

Latogurski VI (1979) Rocognizing of independent population of Antarctic krill. Rybnoe Khoziaistvo 1979, 12-14 (in Russian).

Linnenbrink M, Wang J, Hardouin EA, et al. (2013) The role of biogeography in shaping diversity of the intestinal microbiota in house mice. Mol. Ecol. 22, 1904-1916.

Lozupone C, Knight R (2005) UniFrac: a new phylogenetic method for comparing microbial communities. Appl. Environ. Microbiol. 71, 8228-8235.

Mackintosh NA (1973) Distribution of post-larval krill in the Antarctic. Discov. Rep. 36, 95-156.

McDonald D, Price MN, Goodrich J, et al. (2012) An improved Greengenes taxonomy with explicit ranks for ecological and evolutionary analyses of bacteria and archaea. ISME J. 6, 610-618. 
Miller ET, Svanback R, Bohannan BJM (2018) Microbiomes as metacommunities: understanding hostassociated microbes through metacommunity ecology. Trends Ecol. Evol. 33, 926-935.

Moodley Y, Linz B, Yamaoka Y, et al. (2009) The peopling of the Pacific from a bacterial perspective. Science 323, 527-530.

Nakagawa S, Schielzeth H (2013) A general and simple method for obtaining R2 from generalized linear mixed-effects models. Methods in Ecology and Evolution 4, 133-142.

Nicol S, Foster J (2016) The fishery for Antarctic Krill: Its current status and management regime. In: Biology and Ecology of Antarctic Krill. (ed. Siegel V), pp. 387-421. Springer, Cham.

Nowacek DP, Friedlaender AS, Halpin PN, et al. (2011) Super-aggregations of krill and humpback whales in Wilhelmina Bay, Antarctic Peninsula. PLoS One 6, e19173.

Orsi AH, Whitworth T, Nowlin WD (1995) On the meridional extent and fronts of the Antarctic Circumpolar Current. Deep-Sea Res. Pt. 1 Oceanogr. Res. Pap. 42, 641-673.

Peijnenburg KT, Goetze E (2013) High evolutionary potential of marine zooplankton. Ecol. Evol. 3, 2765-2781.

Price MN, Dehal PS, Arkin AP (2010) FastTree 2 - Approximately Maximum-Likelihood Trees for Large Alignments. PLOS ONE 5, e9490.

Reiss CS (2016) Age, growth, mortality, and recruitment of Antarctic Krill, Euphausia superba. In: Biology and Ecology of Antarctic Krill. (ed. V. S), pp. 101-144. Springer, Cham.

Row JR, Knick ST, Oyler-McCance SJ, Lougheed SC, Fedy BC (2017) Developing approaches for linear mixed modeling in landscape genetics through landscape-directed dispersal simulations. Ecol. Evol. 7, 3751-3761.

Segata N, Izard J, Waldron L, et al. (2011) Metagenomic biomarker discovery and explanation. Genome Biol. 12, R60.

Siegel V, Watkins JL (2016) Distribution, biomass and demography of Antarctic Krill, Euphausia superba. In: Biology and Ecology of Antarctic Krill (ed. Siegel V), pp. 21-100. Springer, Cham.

Stegen JC, Lin X, Fredrickson JK, et al. (2013) Quantifying community assembly processes and identifying features that impose them. ISME J. 7, 2069-2079.

Stegen JC, Lin X, Fredrickson JK, Konopka AE (2015) Estimating and mapping ecological processes influencing microbial community assembly. Front. Microbiol. 6, 370.

Tarling GA, Cuzin-Roudy J (2008) External parasite infestation depends on moult-frequency and age in Antarctic krill (Euphausia superba). Polar Biol. 31, 121-130.

Tarling GA, Klevjer T, Fielding S, et al. (2009) Variability and predictability of Antarctic krill swarm structure. Deep-Sea Res. Pt. 1 Oceanogr. Res. Pap. 56, 1994-2012.

Thompson KF, Patel S, Baker CS, Constantine R, Millar CD (2016) Bucking the trend: genetic analysis reveals high diversity, large population size and low differentiation in a deep ocean cetacean. Heredity 116, 277-285.

Van Strien MJ, Keller D, Holderegger R (2012) A new analytical approach to landscape genetic modelling: least-cost transect analysis and linear mixed models. Mol Ecol 21, 4010-4023.

Virtue P, Kawaguchi S, Mclvor J, et al. (2010) Krill growth and condition in Western Indian Ocean sector of the Southern Ocean $30-80^{\circ} \mathrm{E}$ in austral summer 2006. Deep-Sea Res. Pt. II. Top. Stud. Oceanogr. 57, 948-955.

Wang Q, Garrity GM, Tiedje JM, Cole JR (2007) Naive Bayesian classifier for rapid assignment of rRNA sequences into the new bacterial taxonomy. Appl. Environ. Microbiol. 73, 5261-5267.

Ward RD, Woodwark M, Skibinski DOF (1994) A comparison of genetic diversity levels in marine, freshwater, and anadromous fishes. J. Fish Biol. 44, 213-232.

Wirth T, Wang X, Linz B, et al. (2004) Distinguishing human ethnic groups by means of sequences from Helicobacter pylori: lessons from Ladakh. Proc. Natl. Acad. Sci. USA 101, 4746-4751.

Yang C, Cui Y, Didelot X, Yang R, Falush D (2019) Why panmictic bacteria are rare. bioRxiv, 385336.

Yilmaz P, Parfrey LW, Yarza P, et al. (2014) The SILVA and "All-species Living Tree Project (LTP)" taxonomic frameworks. Nucleic Acids Res. 42, D643-D648. 
591

592

593

594

595

596

597

598

599
Zuur AF, leno EN, Elphick CS (2010) A protocol for data exploration to avoid common statistical problems. Methods in Ecology and Evolution 1, 3-14.

Zwirglmaier K, Reid WD, Heywood J, et al. (2015) Linking regional variation of epibiotic bacterial diversity and trophic ecology in a new species of Kiwaidae (Decapoda, Anomura) from East Scotia Ridge (Antarctica) hydrothermal vents. MicrobiologyOpen 4, 136-150.

\section{Author contributions}

LC, LS, RK, and BD conceived the experiments and collected the samples. $L C, A B$ and SB performed the data analysis. LC wrote the manuscript. All authors contributed to discussing results and the final version of the manuscript.

\section{Competing Interests}

The authors declare no competing interests. 
a

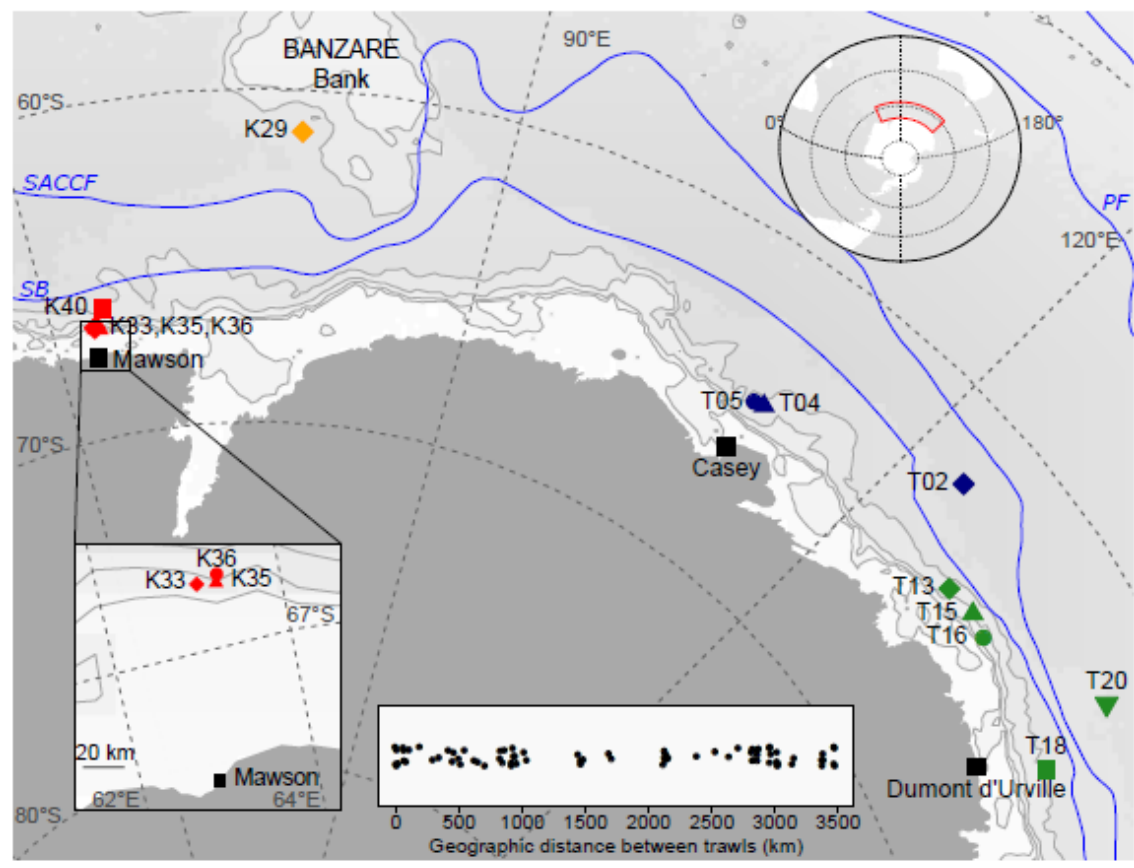

b
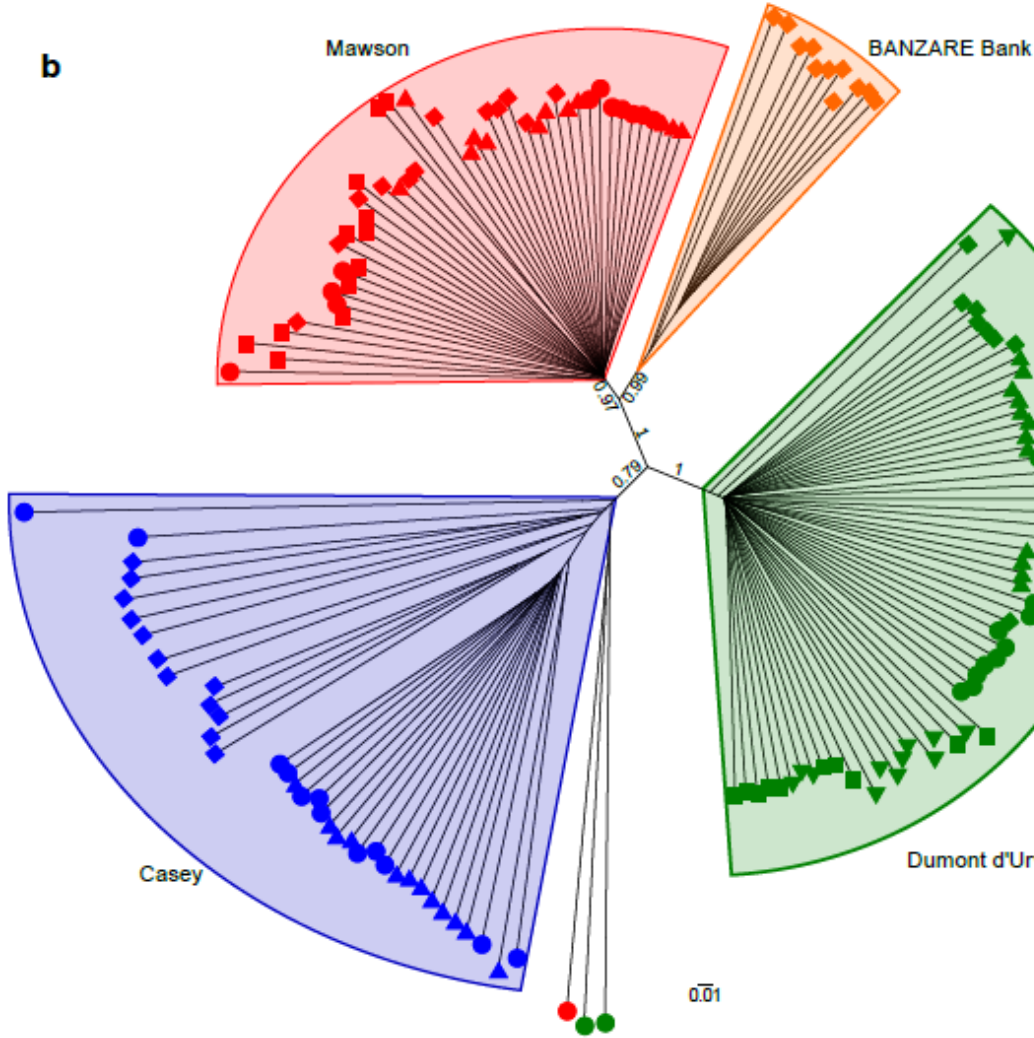

Fig. 1. Spatial structuring of krill moult bacterial communities. a Map of Southern Ocean trawl locations off the East Antarctic coast used to study krill-associated bacteria. Trawls are coloured according to clustering in (b). The inset shows three trawls near Mawson station separated by 4-11 $\mathrm{km}$. Mean locations of the principal fronts (following Orsi et al. 1995) are shown as blue lines. PF Polar Front, SACCF - southern Antarctic Circumpolar Current front, SB - Southern Boundary of ACC. $\mathbf{b}$ UPGMA clustering of krill moult bacterial communities based on unweighted UniFrac distance. Trawls within each region are differentiated by symbols. Jack-knife support is shown for central branches. 
a

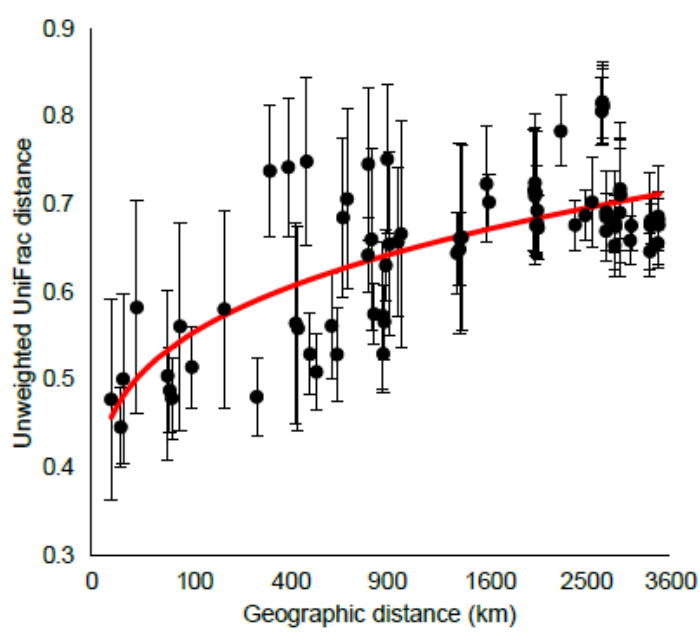

b

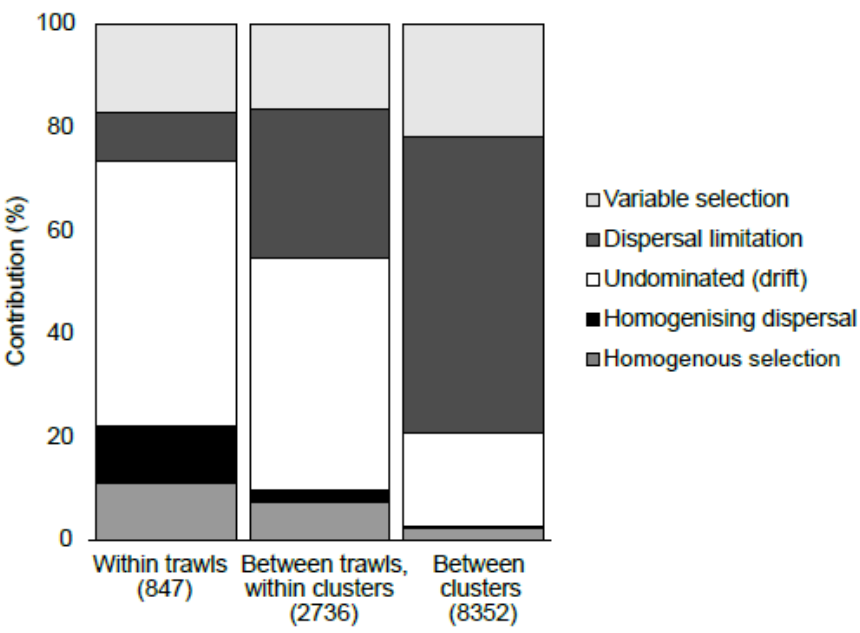

610 Fig. 2. Krill microbiome differentiation driven by dispersal limitation over large geographic

611 distances. a Geographic vs. ecological (unweighted UniFrac) distance for krill moult bacterial

612 communities from separate trawls. The x-axis has been square-root transformed for clarity. Equation

613 of the fitted line: $y=1 /(2.354-0.116 * \log (x))$, adjusted $R^{2}=0.432, p<2 \times 10^{-16}$. Values are means \pm

614 SD unweighted UniFrac distance for all samples from each pair of trawls. b Contribution of ecological

615 processes to krill moult bacterial community assembly at different geographical scales. The number

616 of pairwise comparisons for each category is shown in brackets. 


\section{a. Krill-associated bacteria}

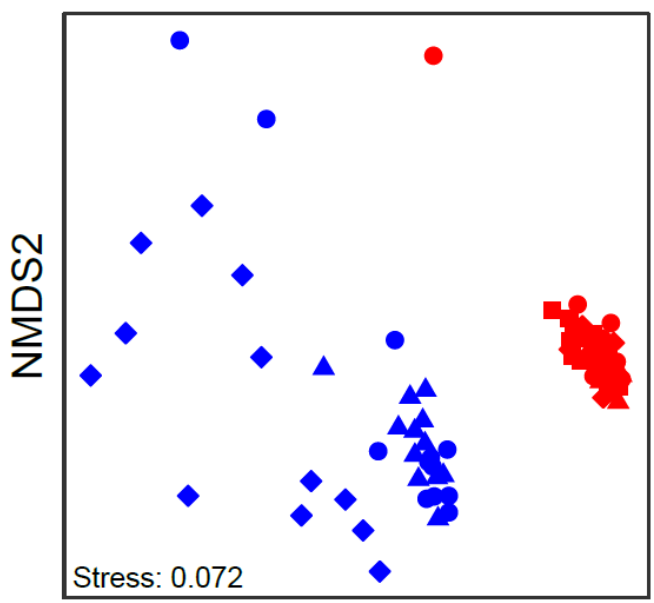

617 1. $85 \%$ or more moult samples).

\section{b. Krill SNP data}

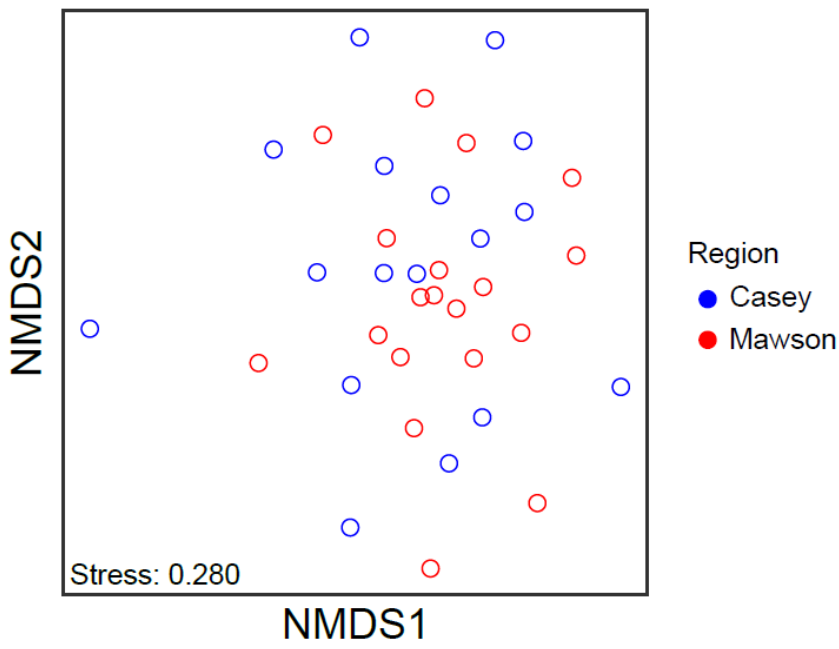

Fig. 3. Spatial structuring of krill microbiomes contrasted with lack of structure observed in SNP markers. Non-metric multidimensional scaling (nMDS) plots for krill from the vicinity of Mawson and Casey stations based on either (a) moult bacterial communities using unweighted UniFrac distance, or (b) Single Nucleotide Polymorphism (SNP) markers using Bray-Curtis dissimilarity of allelic sequence counts (data from Deagle et al. 2015). Krill-associated bacterial community data is from 3 and 5 trawls from the Casey and Mawson regions, respectively, coloured by bacterial geographic cluster as per Fig.

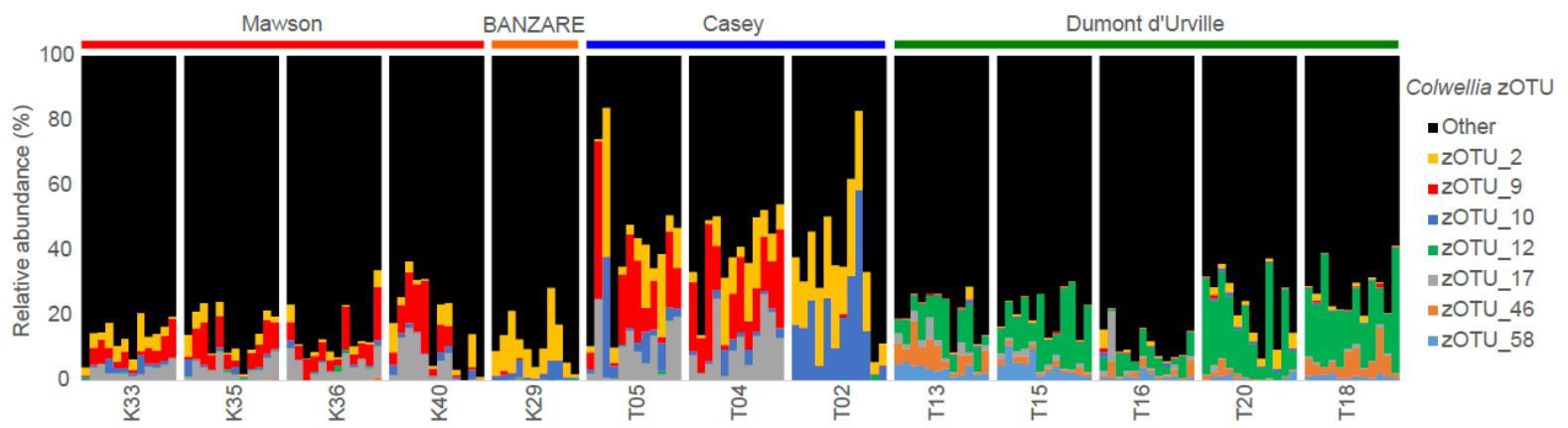

Fig. 4. Relative abundance of dominant Colwellia zOTUs in krill moults shown relative to all other zOTUs, highlighting variability between swarms within a single genus. Samples are grouped by trawl and arranged west to east. Each of the seven zOTUs are members of the core microbiome (present in 
a Weighted UniFrac

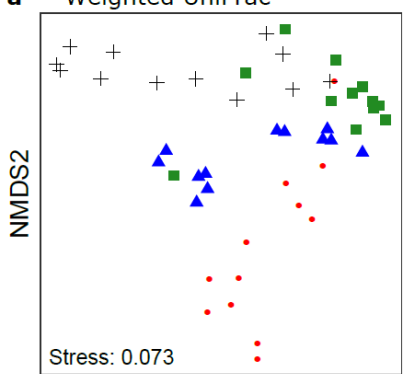

NMDS1 b

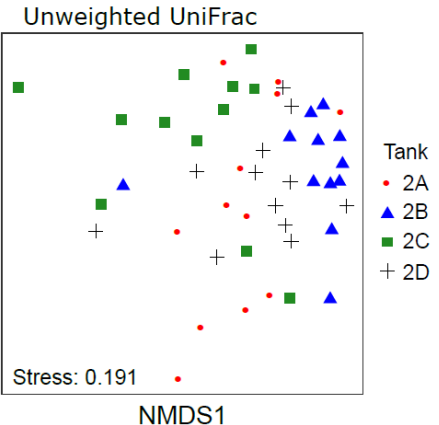

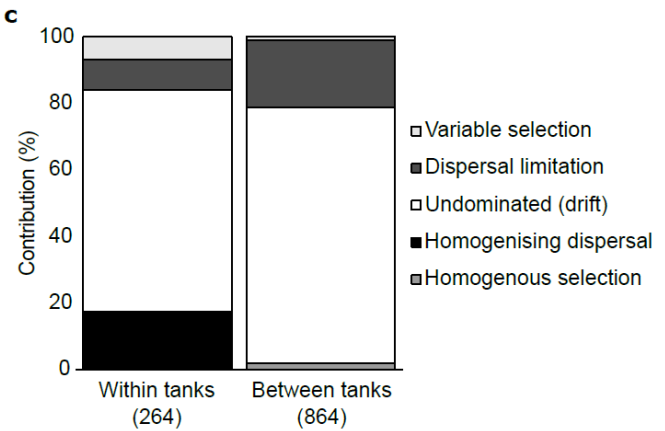

633

634 Fig. 5. Differences between krill bacterial communities originating from a single swarm split across

635 four aquarium tanks for seven months. Non-metric multidimensional scaling (nMDS) plot based on

636 weighted (a) or unweighted UniFrac distance (b). c Contribution of ecological processes to krill

637 epibiont bacterial community assembly within versus between aquarium tanks. The number of

638 pairwise comparisons for each category is shown in brackets.

639

640 